\title{
Hydriding Process in Fe-Ti Alloys Consisting of the FeTi and $\beta$ Ti Phases
}

\author{
By Muneyuki Amano*, Toshiya Hirata*, \\ Takashi Kimura* and Yasuo Sasaki*
}

\begin{abstract}
The hydriding process of $\mathrm{Fe}-\mathrm{Ti}$ alloys has been investigated by means of a volumetric method, differential scanning calorimetry, electron probe X-ray microanalysis and Auger electron spectroscopy. It was found that the hydrogenation became easier with increasing density of phase boundaries between the FeTi and $\beta$ Ti phases and the hydrogenation of the FeTi phase started from the phase boundaries. It is concluded that the air exposed alloys consisting of the FeTi and $\beta$ Ti phases are hydrogenated mainly by a mechanism of phase boundary diffusion of hydrogen atoms in the initial stage of hydriding process.
\end{abstract}

(Received April 9, 1984)

Keyzeords: hydriding process, iron-titanium alloys, hydrogenation, iron titanium phase, $\beta$ titanium phase, phase boundary diffusion

\section{Introduction}

The intermetallic compound $\mathrm{FeTi}$ is one of the most promising candidates for hydrogen storage applications. The difficulty of activation for the first hydrogenation of FeTi was a large problem. This difficulty can be overcome by partially substituting $\mathrm{Fe}$ in FeTi by other elements such as $\mathrm{Ti}^{(1)(2)}, \mathrm{Mn}^{(2)}$ or $\mathrm{Nb}^{(3)}$, and further improvement for the activation can be obtained by adding the oxide phase ${ }^{(1)(2)(4)}$. It has also been known that the alloys consisting of the FeTi and $\beta$ Ti phases are easily hydrogenated at room temperature after some incubation time without any heat treatment ${ }^{(1)(4)}$ and that the $\beta \mathrm{Ti}$ phase is firstly hydrogenated in such alloys ${ }^{(5)}$.

In this paper we will demonstrate that the phase boundary plays an important role for the first hydrogenation of the air exposed alloys consisting of the FeTi and $\beta \mathrm{Ti}$ phases.

\section{Experimental}

Ingots (50 g each) of $\mathrm{FeTi}, \mathrm{FeTi}_{1.04}, \mathrm{FeTi}_{1.06}$, $\mathrm{FeTi}_{1.08}, \quad \mathrm{FeTi}_{1.13}, \quad \mathrm{FeTi}_{1.22}, \quad \mathrm{FeTi}_{1.27}$, $\mathrm{FeTi}_{1.38}, \mathrm{FeTi}_{1.86}, \mathrm{FeTi}_{2.33}$ and $\mathrm{FeTi}_{4}$ were prepared by arc-melting electrolytic iron of

* National Research Institute for Metals, Nakameguro, Tokyo 153, Japan.
$99.9 \%$ and sponge titanium of $99.8 \%$ in a water-cooled copper hearth in an argon atmosphere. The contents of titanium and iron in the alloys were analyzed by chemical analyses, and the phase determination was carried out using a scanning electron microscope equiped with an energy dispersive $\mathrm{X}$-ray analysis system and an electron probe X-ray microanalyzer. The analysis of oxygen in FeTi and $\mathrm{FeTi}_{1.13}$ was carried out using the inert fusion analysis, and the oxygen contents were about $0.06 \mathrm{mass} \%$ in both alloys. The depth profile of each composition in the samples before hydrogenation was obtained using Auger electron spectroscopy.

The rate of hydrogenation was calculated from the change in pressure after introducing hydrogen of $6 \mathrm{MPa}$ to a closed system which had about $15 \mathrm{~cm}^{3}$ inner volume and in which $1 \mathrm{~g}$ sample of -100 mesh was set. The differential scanning calorimetry was carried out under hydrogen atmosphere of $3 \mathrm{MPa}$.

Samples for surface analyses were prepared by cutting ingots to plates $1 \mathrm{~mm}$ thick, which were polished using emery paper and $\mathrm{Al}_{2} \mathrm{O}_{3}$ powder. Plates of $\mathrm{FeTi}_{1.13}\left(5 \times 10 \times 1 \mathrm{~mm}^{3}\right)$ were kept for various periods of time under a hydrogen atmosphere of $6 \mathrm{MPa}$ at $298 \mathrm{~K}$, and the hydriding process was observed using an electron probe X-ray analyzer. Hydrogen gas of seven nine purity was used in this work. 


\section{Experimental Results}

Figure 1 shows the rate of hydrogen absorption at $298 \mathrm{~K}$ and under the initial hydrogen pressure of $6 \mathrm{MPa}$ for the samples of $\mathrm{FeTi}$, $\mathrm{FeTi}_{1.04}, \mathrm{FeTi}_{1.13}, \mathrm{FeTi}_{1.27}$ and $\mathrm{FeTi}_{1.38}$, which were pulverized in air to -100 mesh. The sample of stoichiometric FeTi was not hydrogenated under the condition. On the other hand, the samples of titanium-rich alloys were hydrogenated after some incubation time. The incubation times of the samples of FeTi ${ }_{1.13}$, $\mathrm{FeTi}_{1.27}$ and $\mathrm{FeTi}_{1.38}$, which contain the FeTi and $\beta$ Ti phases are shorter than that of $\mathrm{FeTi}_{1.04}$ consisting only of the FeTi phase ${ }^{(1)}$. It is to be noted that the incubation time decreases and then increases with increasing titanium concentration, as shown in Fig. 1. Another important thing is that even in the incubation time the curves B, C, D and E in Fig. 1 show some slope, i.e., indicating that hydrogenation occurs slowly.

Figure 2 shows a representative differential scanning calorimetry curve of $\mathrm{FeTi}_{1.22}$ pulverized to -12 to +24 mesh in air. The ordinate shows the relative degree of exothermic or endothermic reaction per unit time. The saw-teeth-like exothermic peaks suggest that

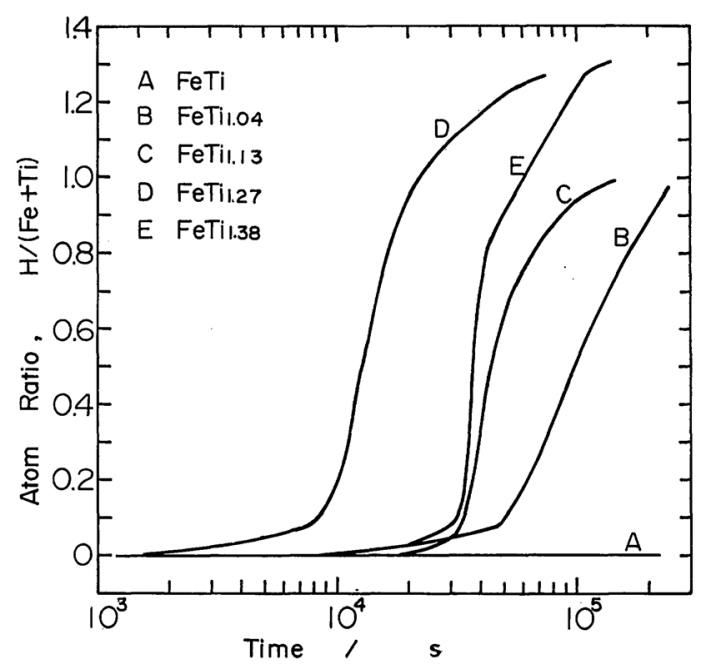

Fig. 1 Amount of hydrogen absorbed vs reaction time at $298 \mathrm{~K}$ for $\mathrm{FeTi}, \mathrm{FeTi}_{1.04}, \mathrm{FeTi}_{1.13}, \mathrm{FeTi}_{1.27}$ and $\mathrm{FeTi}_{1.38}$. The initial hydrogen pressure and particle size are $6 \mathrm{MPa}$ and -100 mesh, respectively.

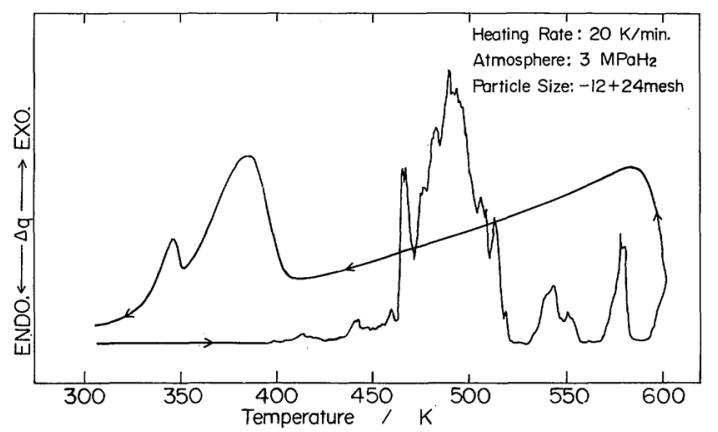

Fig. 2 Differential scanning calorimetry curve of $\mathrm{FeTi}_{1.22}$.

the hydriding occurs accompanied with the formation of cracks. The temperature, shape and number of the peaks changed extremely with the change in particle size. A single peak was observed around $420 \mathrm{~K}$ for the $\mathrm{FeTi}_{1.22}$ sample pulverized to -200 mesh in air and set in the calorimeter within ten minutes. In such a sample of small particles the temperature of the peak shifted to a higher temperature with increasing time of exposure in air. On the other hand, any large difference in the exothermic peaks could not be observed between the samples of -12 to +24 mesh after exposing in air for various times.

In the cooling curve in Fig. 2 the exothermic peaks centered around $383 \mathrm{~K}$ and $340 \mathrm{~K}$ are seen, and they are attributed to the formation of the $\beta$ and $\gamma$ hydrides, respectively. The cooling rate was not constant because of natural cooling.

Figure 3 shows differential scanning calorimetry heating curves of Fe-Ti alloys which contain 50 to 80 atomic percent titanium. Any peak due to hydrogen absorption cannot be seen for the samples of the stoichiometric FeTi. Small exothermic peaks are seen above $410 \mathrm{~K}$ for the samples of $\mathrm{FeTi}_{1.04}$ and $\mathrm{FeTi}_{1.08}$, although the $\beta$ Ti phase could not be observed in these alloys by scanning electron microscopy. Alloys containing titanium more than 53 atomic percent consist of the FeTi and $\beta \mathrm{Ti}$ phases. The degree of exothermic reaction in the temperature range between $400 \mathrm{~K}$ and $600 \mathrm{~K}$ increased initially and then decreases with increasing titanium concentration. The samples of $\mathrm{FeTi}_{2.33}$ which contained the maximum 


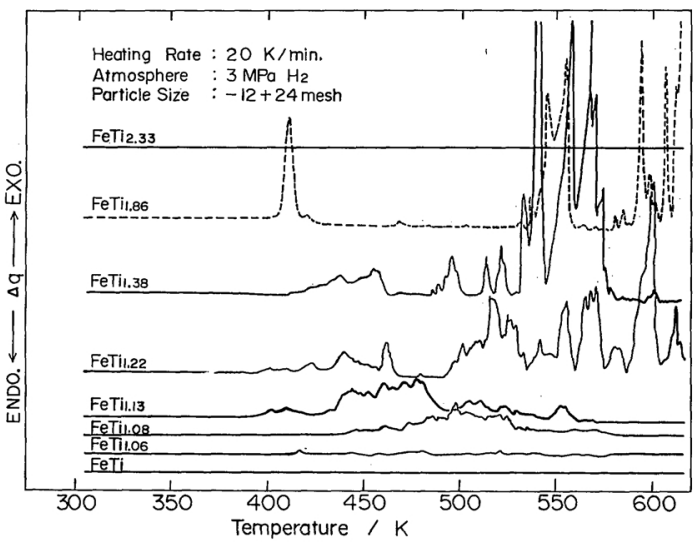

Fig. 3 Differential scanning calorimetry curves of FeTi alloys.

amount of $\beta \mathrm{Ti}$ phase did not react with hydrogen, as shown in Fig. 3.

Figure 4 shows a representative depth profile obtained by Auger electron microscopy for $\mathrm{FeTi}_{4}$ consisting only of the $\beta \mathrm{Ti}$ phase. The sputtering rate was $0.17 \mathrm{~nm} / \mathrm{s}$. The result shows that the region of high oxygen concentration is fairly deep, i.e., about $20 \mathrm{~nm}$. The high carbon concentration on the surface is considered to be due to adsorption of $\mathrm{CO}$. The contents of oxygen and carbon seem to be fairly high even after sputtering of several hundred seconds, however it is considered to be mainly caused by the adsorption of $\mathrm{CO}$ during the measurement, because these values became very small when the measurement was carried out during sputtering. In case of stoichiometric FeTi the depth of high oxygen region was about $6 \mathrm{~nm}$

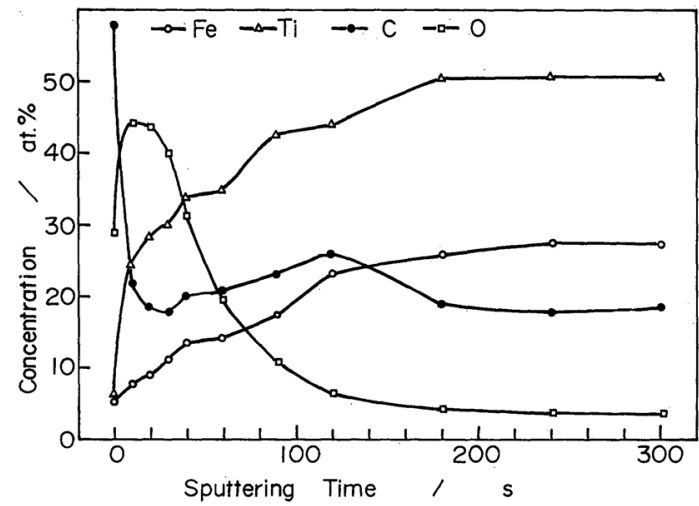

Fig. 4 Auger electron spectroscopy depth profiles of $\mathrm{FeTi}_{4}$ (sputtering rate; $0.17 \mathrm{~nm} / \mathrm{s}$ ). and much smaller than that of $\beta$ Ti.

Figure 5 shows a backscattering electron image of the plate of $\mathrm{FeTi}_{1.13}$ which was partially hydrogenated by keeping it for $108 \mathrm{ks}$ in hydrogen of $6 \mathrm{MPa}$ at $298 \mathrm{~K}$. The black region is the $\beta \mathrm{Ti}$ phase which forms network. The small precipitates in the $\beta$ Ti phase are the secondary precipitates of the FeTi and oxide phases, which will be described in more detail later. As shown in this image, the surface relief is seen in the FeTi phase along both the sides of the phase boundary, and many cracks are also seen in the FeTi phase.

Figure 6 shows a backscattering electron image of the plate of $\mathrm{FeTi}_{1.13}$ whose surface was hydrogenated completely by keeping it for $252 \mathrm{ks}$ in hydrogen of $6 \mathrm{MPa}$ at $298 \mathrm{~K}$.

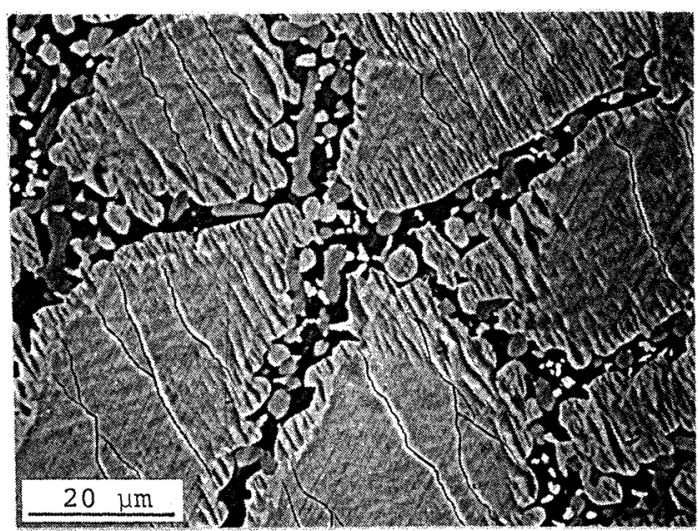

Fig. 5 A backscattering electron image of the plate of $\mathrm{FeTi}_{1.13}$ which was partially hydrided in hydrogen of $6 \mathrm{MPa}$ at $298 \mathrm{~K}$.

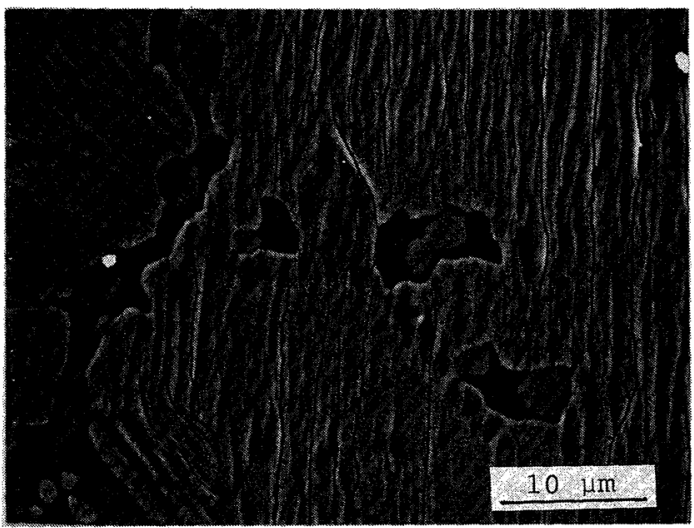

Fig. 6 A backscattering electron image of the plate of $\mathrm{FeTi}_{1.13}$ which was hydrided in hydrogen of $6 \mathrm{MPa}$ at $298 \mathrm{~K}$. 


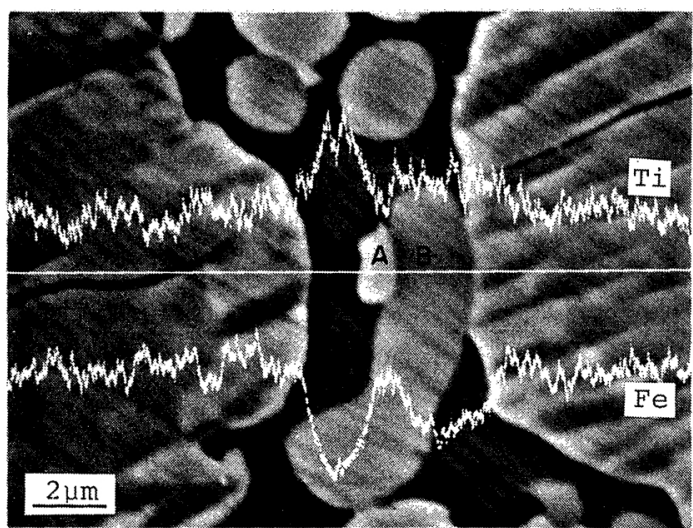

Fig. 7 Line analysis of $\mathrm{FeTi}_{1.13}$ using an electron probe X-ray microanalyzer.

Surface relief and cracks are considered to be generated mainly by the decomposition of the hydride of FeTi phase during decreasing hydrogen pressure to $0.1 \mathrm{MPa}$ before taking out the plate from the apparatus, because tension stress in the FeTi phase is generated at that time. On the other hand, any surface relief cannot be seen in the $\beta$ Ti phase, as shown in Figs. 5 and 6 , although the $\beta$ Ti phase is considered to be hydrogenated firstly. It is attributed to the stableness of the $\beta$ Ti hydride which can hardly decompose at room temperature.

Figure 7 shows the result of line analysis of the small precipitates in the $\beta \mathrm{Ti}$ phase in $\mathrm{FeTi}_{1.13}$. The smaller precipitate denoted as $\mathrm{A}$ is the FeTi phase, and the larger precipitate denoted as $\mathbf{B}$ is the oxide phase whose composition can be expressed as about $\mathrm{FeTi}_{1.6} \mathrm{O}_{0.4}$. These precipitates are considered to be formed during the eutectic transformation.

\section{Discussion}

It has been anticipated before the present work that the hydrogenation of Fe-Ti alloys becomes easier with increasing $\beta$ Ti phase because the $\beta$ Ti phase is reported to be hydrogenated firstly ${ }^{(5)}$. However, it is clarified in this work that there is an optimum composition range for easy hydrogenation in the alloy samples prepared in air as shown in Figs. 1 and 3. These results suggest that the hydrogenation of $\mathrm{Fe}-\mathrm{Ti}$ alloys becomes easier with increasing density of phase boundaries between the FeTi and $\beta \mathrm{Ti}$ phases. If the surface of the $\beta \mathrm{Ti}$ phase is clean, the $\beta$ Ti phase will be hydrided from its surface. However, the surface of the $\beta$ Ti phase is usually covered with a thick oxide film, as shown in Fig. 4. It is, therefore, considered that the penetration of hydrogen atoms through the phase boundaries plays a dominant role for the first hydrogenation of air exposed alloys consisting of the FeTi and $\beta$ Ti phases. This prediction could be confirmed by the direct observation of the hydriding process, as shown in Fig. 5, i.e., the hydrogenation of the FeTi phase started from the phase boundaries. The rate of hydrogenation was very slow for the bulk specimen. It is attributed to the small diffusion coefficient of hydrogen in the hydride of the FeTi phase at room temperature, i.e., about $(1.2 \pm 0.8) \times 10^{-16} \mathrm{~m}^{2} / \mathrm{s}$ at $300 \mathrm{~K}$ for $\mathrm{FeTiH}_{1.03}{ }^{(6)}$.

Figure 8 shows a schematic explanation of the initial stage in the hydriding process for an alloy consisting of the FeTi and $\beta \mathrm{Ti}$ phases. It is not clear at present what operates as a catalyst for the dissociation of hydrogen molecules. As the possible catalysts, iron clusters which are formed by surface segregation ${ }^{(7)}$, $\mathrm{FeTi}^{(8)}$ and surface oxide ${ }^{(9)-(11)}$ have been proposed. If cracks in the oxide film are formed on the interface between hydrogenated and nonhydrogenated regions in the FeTi phase, hydrogen atoms will be able to penetrate also from the route 2 denoted in Fig. 8.

Although $\mathrm{FeTi}_{1.06}$ and $\mathrm{FeTi}_{1.08}$ consist only of the FeTi phase, the hydrogenation of these samples was much easier than that of the stoichiometric FeTi. These samples showed exothermic peaks above $410 \mathrm{~K}$, although they

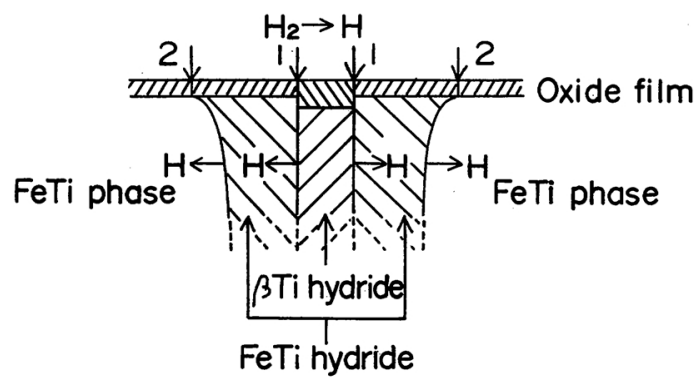

Fig. 8 A schematic explanation of the initial stage in the hydriding process for an alloy consisting of the FeTi and $\beta$ Ti phases. 
were small compared with those of samples containing the $\beta$ Ti phase as shown in Fig. 5 . This result suggests that microscopically distributed titanium rich regions act similarly as the $\beta$ Ti phase, though any phase boundary does not exist, therefore, hydrogen atoms cannot penetrate into the alloy so easily as the case of the phase boundary between the FeTi and $\beta$ Ti phases.

In conclusion, the air exposed alloys consisting of the FeTi and $\beta$ Ti phases are hydrogenated by a mechanism of phase boundary diffusion of hydrogen atoms in the initial stage of the first hydrogenation.

\section{Acknowledgement}

The authors are grateful to Dr. K. Yoshihara for the assistance in Auger electron spectroscopy and for the valuable discussion.

\section{REFERENCES}

(1) M. Amano and Y. Sasaki: Trans. JIM, Suppl., 21 (1980), 329.

(2) G. D. Sandrock, J. J. Reilly and J. R. Johnson: Proc. 11th Intersociety Energy Conversion Engineering Conference, AIChE, (1976), p. 965.

(3) Y. Sasaki and M. Amano: Hydrogen Energy Progress, ed. by T. N. Veziroglu et al., Pergamon, Oxford, (1980), p. 891.

(4) M. Amano, Y. Sasaki and T. Yoshioka: J. Japan Inst. Metals, 45 (1981), 957 (in Japanese).

(5) T. Matsumoto, M. Amano and Y. Sasaki: J. Less-Common Met., 45 (1982), 443

(6) R. C. Bowman, Jr. and W. E. Tadlock: Solid State Comm., 32 (1979), 313.

(7) L. Schlapbach and T. Riesterer: Appl. Phys., A32 (1983), 169.

(8) P. S. Rudman: J. Less-Common Met., 89 (1983), 93.

(9) K. Hiebl, E. Tuscher, H. Bittner: Monatsh. Chemie, 110 (1979), 9.

(10) T. Matsumoto and M. Amano: Scripta Met., 15 (1981), 879.

(11) T. Schober and D. G. Westlake: Scripta Met., 15 (1981), 913. 